Revue

de Sémantique

et Pragmatique
Revue de Sémantique et Pragmatique

37 | 2015

Sémantique et pragmatique

\title{
Comment le préverbe traduit l'espace (sur l'exemple des correspondants polonais du verbe tomber)
}

Joanna Cholewa

\section{(2) OpenEdition}

Journals

Édition électronique

URL : http://journals.openedition.org/rsp/970

DOI : $10.4000 /$ rsp. 970

ISSN : 2610-4377

Éditeur

Presses universitaires d'Orléans

Édition imprimée

Date de publication : 1 juin 2015

Pagination : 25-36

ISSN : 1285-4093

Référence électronique

Joanna Cholewa, «Comment le préverbe traduit l'espace (sur l'exemple des correspondants polonais du verbe tomber) », Revue de Sémantique et Pragmatique [En ligne], 37 | 2015, mis en ligne le 01 juin 2016, consulté le 24 juin 2020. URL : http://journals.openedition.org/rsp/970 ; DOI : https://doi.org/ $10.4000 /$ rsp.970

Ce document a été généré automatiquement le 24 juin 2020.

Revue de Sémantique et Pragmatique 


\section{Comment le préverbe traduit l'espace (sur l'exemple des correspondants polonais du verbe tomber)}

Joanna Cholewa

Une partie importante de verbes en polonais est formée à l'aide de préverbes, qui se combinent avec différentes bases verbales, le même préverbe pouvant se lier avec plusieurs bases et la même base sélectionner divers préverbes. D'un côté, il y a donc dopaść (atteindre, attraper, se jeter sur), do-powiedzieć (ajouter à ce qu'on a dit), do-dać (ajouter, additionner), do-puścić (admettre), et de l'autre do-paść (attrapper, atteindre, se saisir de), przy-paść (tomber, survenir), na-paść (attaquer, tomber, fondre sur), od-paść (tomber de, se détacher), où la base verbale -paść correspond au verbe français tomber. La distribution des préverbes suivant les bases verbales obéit à des contraintes : il faut que les traits sémantiques postulés par la base du verbe acceptent ceux que contient le préverbe (Przybylska, 2006). Chacun des préverbes polonais a plusieurs nuances de sens. Certains auteurs cherchent, dans le cadre de la recherche en linguistique cognitive, leurs invariants. Ainsi, Przybylska décrit les 'superschèmes' de quelques préverbes polonais : elle propose pour chacun d'eux plus d'une dizaine de sens, rassemblés sous un invariant abstrait, s'actualisant dans un contexte, créé par la base verbale donnée, ainsi qu'un schéma syntaxique. D'autres auteurs (Janowska, 1999 ; Kudra, 1983) préfèrent, comme le font les dictionnaires, décrire le sens principal, apparaissant dans la plupart des formations avec un préverbe donné, et les sens secondaires. Dans la suite, nous allons présenter quelques-uns des préverbes polonais, en nous servant des correspondants du verbe français tomber. Il s'avère en effet qu'un grand nombre de ceux-ci sont des dérivés du verbe polonais paść/padać, qui a dans son sémantisme, comme le verbe français tomber, les éléments de sens d'orientation verticale et de direction négative (Cholewa, 2011). D'après Vandeloise (1986) et Borillo (1998), il est possible d'évoquer l'orientation verticale quand un verbe de mouvement décrit le déplacement de la cible (objet à localiser) selon l'axe vertical, la direction 
négative étant donné par la terre. Puisqu'en polonais la même base verbale traduit différents emplois de tomber en sélectionnant les préverbes différents, on peut s'attendre à ce que chacun des préverbes y ajoute un trait sémantique supplémentaire. Nous n'avons pas l'ambition de présenter les sens des préverbes dont il sera question dans la suite dans toute leur ampleur. En effet, la recherche d'un invariant éventuel de chacun exigerait un travail séparé (travail qu'a déjà partiellement entrepris Przybylska, 2006, en décrivant les préverbes do-, od-, prze-, roz- et $u$-). Le rôle des fragments de définitions cités, décrivant le sens des préverbes en polonais, ne sera qu'auxiliaire : celles-ci témoignent de la direction des recherches qui ont été faites dans le domaine. Nous avons volontairement choisi pour notre analyse les emplois de tomber qui décrivent le mouvement dans l'espace, laissant de côté nombre d'acceptions abstraites, où sont conceptualisés l'assujettissement (tomber sous le coup de la loi), la dégradation de l'état physique (tomber de fatigue) ou de l'état psychique de la cible (tomber dans une dépression), la diminution de l'intensité (sa colère est tombée), et autres. Notre objectif est d'abord de comparer les emplois sélectionnés du simplex français avec ceux des préverbés polonais qui lui correspondent, ensuite d'observer quelle est l'information sémantique que les préverbes choisis véhiculent en se combinant avec la base paść/ padać, ce qui fait que le polonais dispose d'une variété de préverbés là où le français n'a que la seule forme tomber, et enfin d'observer quels éléments du cotexte ou du contexte expriment cette information sémantique en français.

\section{Formations avec le préverbe $u$ -}

D’après Przybylska (2006), le préverbe $u$ - induit la représentation d'un changement. Elle distingue pour ce préverbe une trentaine de nuances de sens, rassemblées sous l'invariant qu'elle décrit ainsi : dans la situation 1 (avant le changement), il existe un site constituant un ensemble séparé du reste de l'espace. Le préverbe $u$ - impose de focaliser l'attention sur une partie périphérique de cet ensemble (Przybylska, 2006, 232). Le préverbe $u$ - est utilisé avec la base verbale paść/padać dans la traduction de tomber intransitif signifiant 'choir, chuter' (LVF). Pourtant, deux cas distincts sont à envisager, tomber s'utilisant avec ou sans syntagme prépositionnel (SP), pour lesquels les correspondants polonais ne sont pas identiques du point de vue morphologique.

(1) tomber = upaść/padać

Tomber sans circonstant se traduit en polonais par le couple perfectif/ imperfectif upaść/padać:

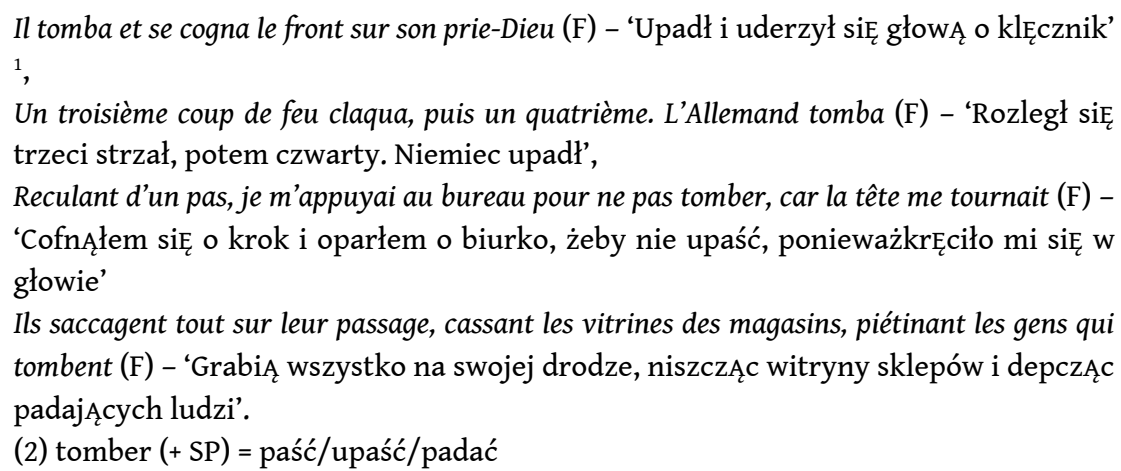

Il n'en est pas de même pour les emplois où tomber est suivi d'un SP, exprimant la direction ou la manière. En effet, le polonais dispose alors de deux formes parallèles 
pour l'aspect perfectif : paść et son dérivé upaść, formé avec le préverbe u-. Ainsi, on traduira :

tomber sur le banc, sur les coussins (F) - 'paść/upaść/padać na ławkĘ, na poduszki', tomber sur un fauteuil - 'paść/upaść/padać na fotel' (WSPF),

tomber à genoux devant qqn - 'paść/upaść/padać na kolana przed kimś' (WSFP),

tomber à la renverse, de toute sa hauteur, la tête la première - 'paść/upaść/padać na

wznak, jak długi, głowĄ w dół' (WSFP).

Il existe d'ailleurs d'autres formations, avec le même préverbe, où la forme préfixale et la forme sans préfixe ont le même sens et s'utilisent l'une pour l'autre :

siąiść/usiąść (s'asseoir) : il s'est assis - '(on) siadł/usiadł',

klęknĄć/uklĘknĄć (s'agenouiller) : il s'est agenouillé - '(on) klęknĄt/uklęknĄl',

kucnĄć/ukucnĄć (s'accroupir) : il s'est accroupi - '(on) kucnĄł/ukucnĄł'.

Przybylska $(2006,233)$ constate que $u$ - est un préverbe 'à deux directions' parce qu'il construit des formations qui peuvent désigner soit le mouvement de la cible de l'intérieur vers l'extérieur (umknAcć, ujść - s'enfuir, ulecieć - s'envoler), soit le mouvement inverse, de l'extérieur vers l'intérieur du site (ukruszyć - ébrécher, ugnieść tasser, ugryźć - mordre, uciąć - couper), en focalisant l'attention sur une partie périphérique d'un lieu. Le 'mouvement' est conceptualisé soit comme un déplacement réel dans l'espace physique, soit comme un mouvement abstrait. Mais, d'après cette auteur (Przybylska, 2006, 272), l'hypothèse que le préverbe $u$ - soit l'indice d'un mouvement physique à orientation verticale, dont témoigne le verbe upaść, mais aussi d'autres verbes déjà cités (uklęlknĄć, ukucnĄć, usiąść), n'est pas vraiment confirmée. A son avis, le lien entre $u$ - et le mouvement à orientation verticale résulte plutôt d'implications contextuelles isolées, ce trait sémantique n'entrant pas dans le sémantisme du préverbe en question. À notre avis, il n'est pas nécessaire de chercher dans le sens du préverbe $u$ - d'indices de mouvement suivant l'axe vertical car ce trait sémantique est déjà exprimé par les bases verbales des verbes en question (u-paść, $u$ $k l E ̨ k n A c ́ c, u-k u c n A q c ́, u$-siĄść) et il n'est pas indispensable que celui-ci répète le même sens. Ajouté à la base paść, le préverbe $u$ - signifie la 'focalisation sur une partie périphérique du lieu de fin de mouvement', même si ce lieu est entièrement inféré, comme dans les exemples évoqués dans (1), où le lieu inféré de fin de mouvement est 'le sol', 'la terre'.

\section{Formations avec le préverbe $z-/ s$ -}

7 Pour montrer le sens que le préverbe $s$ - (forme s'utilisant devant une consonne sourde) ajoute à la base paść/padać, nous allons nous servir également de deux emplois distincts traduisant tomber:

(3) tomber = spaść/padać

8 Le premier correspond à tomber intransitif se référant aux précipitations atmosphériques ('descendre vers le sol', LVF $n^{\circ} 4$ ) : Il tombe de la neige, La neige tombe. Pour l'aspect imperfectif, le polonais utilise dans ce cas le verbe padać:

la neige/la pluie tombe - 'pada śnieg/deszcz' (WSFP), Nous avions bien une tente, mais la pluie s'étant mise à tomber, nous recherchâmes un abri (F) - 'Mieliśmy namiot, ale zacż̨ł padać deszcz i poszukaliśmy schronienia',

La neige tombait, silencieuse, avec des froissements de plume (F) - 'Śnieg padał cicho, ze szmerem ptasich piór',

alors que le perfectif sélectionnera spaść : 
Brusquement une averse était tombée (F) - 'Nagle spadła ulewa/spadł ulewny deszcz',

La pluie tomba, qui fit fondre la neige (F) - 'Spadł deszcz i stopił śnieg',

La neige est tombée encore et Pauline voudrait tant qu'elle fasse aussi sur elle

un chaud manteau d'invisibilité (F) - 'Znowu spadł śnieg i Paulina zapragnĘła mocno,

żeby i na niej zrobił gruby, niewidzialny płaszcz'.

(4) tomber de = spaść/spadać z

Le deuxième cas à étudier est le verbe spaść/spadać $z$, traduisant tomber de dans le sens de 'se détacher' (LVF ${ }^{\circ}{ }^{\circ}$ ). Il est utilisé avec No [+humain] ou [+concret] et implique l'existence du site - entité qui n'a pas d'intérieur, avec laquelle la cible est en contact au début du mouvement. Spaść/spadać peut sélectionner un SP de type ablatif ou de type adlatif, avec ellipse du SP ablatif, l'un ou l'autre obligatoire, commençant par une préposition désignant le début du mouvement $(z)$ ou la fin de celui-ci (na/wokót) : tomber suivi d'un SP de type ablatif :

Les feuilles tombent des arbres - 'Liście spadają z drzew' (WSFP),

Je faillis tomber de ma chaise (F) - 'O mało nie spadłem $\mathrm{z}$ krzesła',

Le sac lui était tombé de l'épaule ; il avait lâché son fusil (F) - 'Torba spadła mu z

ramienia ; wypuścił strzelbĘ' ;

11 tomber suivi d'un SP adlatif, avec ellipse du SP ablatif :

Le fruit est tombé à terre - 'Owoc spadł na ziemiĘ' (WSFP),

Les bombes les plus proches sont tombées autour du Sacré-Coeur (F) - 'Najbliższe bomby

spadły wokół Sacré-Coeur'.

Dans le couple spaść/spadać $z$, le préverbe a une valeur sémantique de perte de contact et d'éloignement d'un lieu, désigné par le nom du SP ablatif (des arbres, de ma chaise, de l'épaule). Dans les exemples avec ellipse du SP ablatif, le site avec lequel la cible perd le contact est inféré : le fruit est tombé (de l'arbre) à terre, les bombes les plus proches sont tombées (du ciel, des bombardiers) autour du Sacré-Coeur. Il serait possible de traduire ces exemples en utilisant le préverbé avec le morphème u-, qui a été présenté dans la section précédente :

Le fruit est tombé à terre - 'Owoc upadł na ziemię'

Les bombes les plus proches sont tombées autour du Sacré-Coeur (F) - 'Najbliższe

bomby upadły wokół Sacré-Coeur',

mais cette solution entraînerait un changement sémantique important : disparaitrait alors le sens de 'perte de contact et d'éloignement d'un lieu' et apparaîtrait celui de 'focalisation sur une partie périphérique du lieu de fin de mouvement'.

\section{Formations avec le préverbe 0 -}

14 Le préverbe o- se combine avec la base verbale padać (uniquement à l'aspect imperfectif) pour traduire 'tomber' dans deux sens différents, décrivant le mouvement virtuel.

(5) tomber (sur, à, vers) = opadać (na, do)

Opadać traduit tomber transitif indirect, décrivant le mouvement virtuel effectué par la cible (le mouvement réel étant un balayage visuel fait par le regard de l'observateur), où No [+concret] appartient à la classe 'parties du corps' ou 'tissus' ('pendre sur' dans LVF, $\left.\mathrm{n}^{\circ} 10\right)$ :

ses cheveux lui tombent sur les épaules - 'włosy mu opadają na ramiona' (WSFP), 
C'était un grand drapeau de soie rouge, tombant à plis rigides de toute sa longueur contre le mur (F) - 'to była wielka flaga z czerwonego jedwabiu, która całĄ swoją długościĄ opadała przy ścianie sztywnymi fałdami’,

la jupe tombe aux chevilles (LVF) - 'spódnica opada do kostek'. adlatif), précise le lieu où s'achève le mouvement virtuel. Il est possible d'imaginer aussi, dans ce type d'emplois, le SP qui préciserait le lieu de début de mouvement (la jupe tombe des hanches aux chevilles - 'spódnica opada od bioder do kostek/po kostki'). À ce groupe il faut ajouter des exemples avec No 'lieu géographique' : Le versant sud, donc, tombe vraiment à pic (F) - 'Południowe zbocze opada wiĘc naprawdĘ stromo'. Le verbe peut y être accompagné d'un SP répondant à laquestion 'comment'.

(6) tomber = opadać

Le deuxième cas à envisager est le verbe opadać qui traduit tomber intransitif, signifiant 's'affaisser, s'avachir' (LVF n¹2) :

Ses épaules tombent. (LVF) - 'Ramiona mu opadajĄ. / Ma opadajĄce ramiona.'

Il n'est pas toujours possible de traduire ce sens de tomber par opadać. En effet, Ses joues tombent (exemple cité à côté du précédent dans LVF), se traduira plutôt par 'Ma obwisłe policzki', formation avec le même préverbe mais une base différente (wisieć - pendre). D'après Janowska $(1999,38)$, le préverbe o- signale que l'action expriméepar la base verbale se déroule autour du site: obejść/ obchodzić - faire le tour de, contourner (à pied), objechać/ objeżdżać - faire le tour de, contourner (en voiture), obandażować bander, faire un pansement, opakować - envelopper, emballer, ou bien concerne une partie importante de l'extérieur du site : obić, obttuc - ébrécher. Associé à la base padać, le préverbe o- véhicule un sens différent de celui exprimé aussi bien par la base verbale que par la préposition qui suit le verbe $(n a, d o):$ au sens de déplacement vers le bas (padać) il ajoute l'information que l'action exprimée par la base verbale concerne une grande partie de l'extérieur d'un lieu, et la préposition indique le point ou le déplacement se termine.

\section{Formations avec le préverbe $w$ -}

Le verbe wpaść/wpadać traduit tomber transitif indirect utilisé dans deux emplois distincts, s'utilisant avec deux prépositions : dans et chez.

(7) tomber dans = wpaść/wpadać do/w

Wpaść/wpadać do/w traduit tomber dans, utilisé au sens de 'chuter dans' (LVF n²), avec No [+concret], [+humain] ou [+abstrait]. Les traits sémantiques de la cible n'ont pas d'importance pour la traduction, par contre, No [+concret] semble entraîner automatiquement N1 [+concret] dans le SP, et No [+abstrait] - N1 également [+abstrait] :

Un mascara dans son étui brillant tombe dans son sac (F) - 'Tusz do rzĘs w błyszczĄcym

etui wpada jej do torebki',

Une voiture tombe dans le ravin (F) - 'Samochód wpada do rowu',

Ces mots que je prononçais tombaient dans le silence (F) - 'Wypowiadane przeze mnie słowa wpadały w ciszę',

Plusieurs fois j'ai dormi avec ma grand-mère : on tombe dans un nid de plumes et on rabat sur soi une couverture douce (F) - 'Kilka razy spałem z babciĄ: wpada siદ̨ do puchowego gniazda i nakrywa miĘkkĄ kołdrą'.

(8) tomber chez = wpaść/wpadać do

Revue de Sémantique et Pragmatique, 37| 2015 

avec N0 et N1 [+humain] se traduit en polonais par wpaść/wpadać do (kogoś) : du site, avec le franchissement de la frontière d'un lieu. Les verbes que celui-ci forme entrent en opposition avec ceux créés à l'aide de $w$ - (déplacement vers l'intérieur d'un lieu) : wypaść/wypadać (tomber de) - wpaść/ wpadać (tomber dans), wyjechać (partir) wjechać (entrer), wyprowadzić (faire sortir) - wprowadzić (faire entrer), wynieść (sortir q.ch.) - wnieść (apporter). Ils impliquent l'ablativité et sélectionnent avant tout les SP à direction ablative, mais peuvent aussi être suivis d'un SP adlatif, avec ellipse d'un circonstant ablatif : wyjechać do Londynu - 'partir pour Londres' (Kudra 1983, 50-52). Deux types d'emplois de tomber se traduisent par la base paść/padać avec le préverbe $w y-:$ tomber de transitif indirect et tomber intransitif.

(9) tomber de = wypaść/wypadać z

Tomber de transitif indirect se traduit par wypaść/wypadać z, qui s'emploie avec NO [+concret] ou [+humain], et N1 [+concret], désignant le lieu à l'intérieur duquel la cible (No) se trouve au début du mouvement :

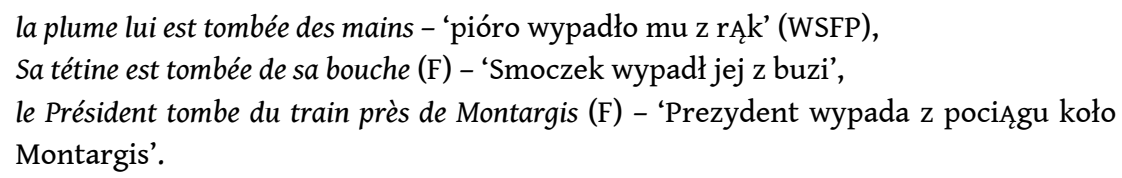

Les exemples qui figurent ici sont assimilables par leur construction à ceux de la section (4) : tomber de + N. La différence entre les deux consiste en caractéristiques du site (lieu où commence le mouvement) : pour (4), ce lieu est une entité à surface plate, sans intérieur, avec laquelle la cible reste en contact temporaire, passager, alors que pour (9), le site est une entité dotée d'un intérieur (les mains qui forment un espace fermé, la bouche, le train). En polonais, ces caractéristiques du site influent sur la combinatoire du verbe : dans les exemples de (4) le polonais sélectionne le préverbe s-, alors que dans (9) c'est le préverbe wy-.

(10) tomber = wypaść/wypadać 

plus souvent par la préposition ablative od : odejść od okna (s'éloigner de la fenêtre), ou par la préposition de même type $z$ : odjechać $z$ domu (quitter la maison). Les dérivés avec le préverbe od- se construisent aussi avec les SP de type adlatif, avec ellipse d'un SP ablatif, introduits par les prépositions adlatives : odejść do swojego pokoju (s'en aller dans sa chambre), odlecieć za morze (s'envoler vers la mer).

(11) tomber = odpaść/odpadać od/z

Tomber intransitif se traduit par wypaść/wypadać, mais il faut remarquer que celui-ci correspond à deux sens de tomber que le dictionnaire LVF analyse séparément:

Les cheveux tombent, ils sont malades (LVF) - 'Włosy wypadajĄ, sĄ chore', Ses cheveux lui tombent depuis qu'elle les teint - 'Włosy jej wypadajĄ (wychodzA) odkĄd je farbuje' (WSFP),

Le plombage d'une dent est tombé (LVF) - 'Plomba wypadła (z zĘba)',

Il m'est tombé une dent - 'Wypadł (wyleciał) mi zĄb' (WSFP).

Dans LVF, la phrase Les cheveux tombent, ils sont malades ('Włosy wypadają, sĄ chore') illustre le sens 'se détacher' ( ${ }^{\circ} 5$ dans LVF), avec les feuilles tombent des arbres, alors que le polonais sélectionne pour ce deuxième sens le verbe spaść/spadać, formé avec le préverbe $s^{-}$, que nous avons analysé au début de cet article. La phrase Le plombage d'une dent est tombé ('Plomba wypadła z zĘba') est à son tour assimilé dans LVF au n ${ }^{\circ} 6:$ Un carreau est tombé du mur ('Płytka odpadła od ściany'), qui sera analysé dans la section suivante, pour illustrer tomber au sens de 'se défaire'. L'emploi de wypaść/wypadać pour 'les cheveux' ou 'les dents' suggère qu'ils se trouvent 'à l'intérieur' au début, et qu'il y a un déplacement vers l'extérieur, même si le SP ablatif est absent. En fait, dans ces exemples il a une ellipse du SP ablatif.

\section{Formations avec le préverbe od-}

Le verbe odpaść/odpadać traduisant tomber intransitif, utilisé avec No [+concret] au sens de 'se défaire' ( $n^{\circ} 6$ dans LVF) est suivi de l'une des prépositions ablatives : od ou $z(e)$, reprenant le sens du préverbe et introduisant l'endroit dont s'éloigne un objet :

Un carreau est tombé du mur (LVF) - 'Płytka odpadła od ściany',

Le plâtre tombe du mur (Internet) - 'Gips (tynk) odpada ze ściany',

Quatre ans de sa vie croulaient comme un de ces pans de glace qui tombent de la

banquise et plongent d'un bloc à la mer (F) - 'Cztery lata życia waliły siĘ tak jak lodowe

bloki, które odpadajĄ/odrywajĄ siĘ od góry lodowej i tonĄ/zanurzajĄ siĘ w morzu'.

Dans le dictionnaire LVF, l'exemple Un carreau est tombé du mur illustre tomber au sens de 'se défaire' et figure avec Le plombage d'une dent est tombé,

qui se traduit en polonais par 'Plomba wypadła z zęba'. Il a été inclus dans (10) pour présenter le sens du préverbe wy-. Or, alors que dans cette dernière phrase tomber de se traduit par wypaść, le préverbe wy-indiquant le déplacement de la cible de l'intérieur d'un lieu vers l'extérieur, avec le franchissement de la frontière de ce lieu, pour la première le polonais sélectionne le préverbe od-('Płytka odpadła od ściany'). od-, utilisé avec paść/padać pour traduire tomber de signifie 'quitter un endroit, considéré comme exerçant un blocage sur la cible, et s'éloigner de celui-ci'. Odpaść/odpadać apparaît si le site n'est pas doté d'un intérieur, ce qui caractérise aussi les exemples de (4), et si la relation qui existe entre la cible et le site avant le mouvement est censée être forte, durable, ce qui distingue les exemples de cette section de ceux de (4). Ainsi, est censée 
être durable, forte la relation entre le mur et les carreaux (ils sont collés au mur pour en faire partie), ou entre le mur et le plâtre. La banquise constitue un tout, et seulement des conditions exceptionnelles peuvent faire qu'un morceau s'en détache.

\section{Conclusion}

Les éléments de sens exprimés en français par le verbe et la préposition qui introduit le SP sont en polonais inclus dans trois type d'éléments : base verbale, préposition et préverbe. Dans le corpus d'analyse que nous avons choisi, les éléments de sens d'orientation verticale et de direction vers le bas sont principalement véhiculés par le verbe tomber en français et par la base verbale paść/padać en polonais. Que le sens de la préposition française est partiellement inclus dans le préverbe en polonais n'est pas un constat nouveau (Giermak-Zielińska 1979, Gwiazdecka 2005). Nous avons essayé dans cet article d'observer dans quelle mesure les six préverbes polonais choisis complètent le sens transmis par la base paść/padać dans les formations préfixales traduisant tomber utilisé sans ou avec SP. Dans le tableau qui suit, nous pouvons voir que les éléments de sens tels que l'éloignement d'un lieu ou de la surface d'un lieu, la perte de contact avec un lieu, le mouvement vers l'intérieur d'un lieu et le mouvement vers l'extérieur d'un lieu sont véhiculés en français par la préposition et en polonais en même temps par la préposition et le préverbe. Le rapprochement jusqu'à la frontière du site s'exprime dans les deux langues par la préposition. Les préverbes polonais qui se distinguent dans notre corpus sont les préverbes $u$ - (focalisation de l'action sur une partie périphérique du lieu de fin de mouvement), o- (focalisation de l'action sur une grande partie d'un lieu) et od- (action de quitter un endroit, considéré comme exerçant un blocage sur la cible et s'éloigner de celui-ci). En effet, d'un côté ce sens n'est pas repris par la préposition en polonais, et de l'autre, il n'est pas présent dans la construction française verbe + préposition. Ceci ne veut évidemment pas dire que les deux éléments en question n'existent pas pour le français : il faudrait les chercher dans le cotexte, dans le 
sens véhiculé par la cible et le site.

\begin{tabular}{|c|c|c|c|c|c|}
\hline & \multicolumn{2}{|c|}{ Français } & \multicolumn{3}{|c|}{ Polonais } \\
\hline Eléments de sens & $\frac{8}{5}$ & 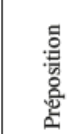 & 荡 & 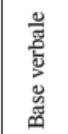 & 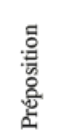 \\
\hline verticalité / soudaineté & tomber & & & $\begin{array}{l}\text { pasćl } \\
\text { padać }\end{array}$ & \\
\hline $\begin{array}{l}\text { rapprochement jusqu'à la frontière du } \\
\text { site ; }\end{array}$ & & \begin{tabular}{|l|} 
sur \\
à \\
vers
\end{tabular} & & & $\begin{array}{l}\text { na } \\
\text { do } \\
\text { ku }\end{array}$ \\
\hline $\begin{array}{l}\text { focalisation sur une partie périphérique } \\
\text { du lieu de fin de mouvement; }\end{array}$ & & & u- & & \\
\hline $\begin{array}{l}\text { éloignement d'un lieu ou de la surface } \\
\text { d'un lieu ; } \\
\text { perte de contact avec un lieu ; }\end{array}$ & & de & $z(s)-$ & & $\begin{array}{l}\mathrm{z}(\mathrm{ze}) \\
\text { od }\end{array}$ \\
\hline $\begin{array}{l}\text { focalisation de l'action sur une grande } \\
\text { partie d'un lieu; }\end{array}$ & & & o- & & \\
\hline $\begin{array}{l}\text { action de quitter un endroit, considéré } \\
\text { comme exerçant un blocage sur la cible, } \\
\text { et de s'éloigner de celui-ci; }\end{array}$ & & & od- & & \\
\hline $\begin{array}{l}\text { mouvement vers l'intérieur d'un lieu avec } \\
\text { le franchissement de la frontière de ce } \\
\text { lieu; }\end{array}$ & & $\begin{array}{l}\text { dans } \\
\text { chez }\end{array}$ & w- & & $\begin{array}{l}w \\
d o\end{array}$ \\
\hline $\begin{array}{l}\text { mouvement de l'intérieur vers l'extérieur } \\
\text { d'un lieu, avec le franchissement de la } \\
\text { frontière de ce lieu ; }\end{array}$ & & de & wy- & & $z$ \\
\hline
\end{tabular}

\section{: $56-65$}

Borillo, A. (1998), L'espace et son expression en français, Paris.

Cholewa, J. (2011), «Espace dans les sens abstraits de quelques verbes de mouvement», Cholewa, J. (2012), «Correspondants polonais du verbe français 'tomber' - emplois locatifs ", Bogacki K., Cholewa J., Rozumko A., Aspects sémantiques et formels dans les recherches linguistiques, Białystok : Wydawnictwo Uniwersytetu w Białymstoku : 45-54. 5 Cholewa, J. (2013), «Le verbe polonais 'paść/padać' et ses dérivés qui traduisent les emplois non locatifs de 'tomber'», Białostockie Archiwum JĘzykowe, 12, Białystok : Wydawnictwo Uniwersytetu w Białymstoku : 11-23.

François J. (et al.), (2007), «Présentation de la classification des Verbes Français de Jean Dubois et Françoise Dubois-Charlier», Langue française, 2007/1 : 3-19.

Giermak-Zielińska, T. (1979), Polskie czasowniki przedrostkowe o znaczeniu przestrzennym i ich odpowiedniki w jĘzyku francuskim, Wrocław.

Gwiazdecka, E. (2005), Aspects, prépositions et préverbes dans une perspective logique et cognitive. Application au polonais: przez/prze-, do/do-, od/od-, thèse de doctorat soutenue en 2005 à Paris IV.

Janowska, A. (1999), Funkcje przestrzenne przedrostków czasownikowych w polszczyźnie, Katowice: Wydawnictwo Uniwersytetu ŚlĄskiego.

40 Kudra, B. (1983), Wpływ przedrostków na łĄczliwość składniowĄ czasowników ruchu, Łódź: Wydawnictwo Uniwersytetu Łódzkiego.

Przybylska, R. (2006), Schematy wyobrażeniowe a semantyka polskich prefiksów czasownikowych do-, od-, prze-, roz-, u-, Kraków: Universitas. 


\section{francuskopolski, t. 1-2, Ciesielska-Borkowska S. et al. (rédaction), Warszawa, Wiedza} Powszechna.

\section{NOTES}

1. Les exemples puisés dans le Frantext ont été traduits par l'auteur de l'article.

\section{RÉSUMÉS}

Comment le préverbe traduit l'espace (sur l'exemple des correspondants polonais du verbe tomber) Les invariants du verbe français tomber et de la base verbale du polonais paść/padać comprennent deux éléments de sens essentiels : l'orientation verticale et la direction négative. Or, la plupart des correspondants polonais de tomber sont des dérivés de paść/padać, formés avec différents préverbes. L'article présente les emplois de tomber et leurs correspondants en polonais, formés avec six préverbes : u-, s-, o-, w-, wy-, od-, qui, se combinant avec la base paść/ padać, ajoutent une information sémantique supplémentaire, différente pour chacun d'eux.

How preverbs express space (using the example of Polish equivalents of French verb tomber) Invariants of French verb tomber and Polish verbal basis paść/padać include two main meaning components: vertical orientation and downstairs direction. Now, most of Polish equivalents of tomber are derived from paść/ padać with differents preverbs. In this paper, we provide a study of six Polish preverbs : $u_{-}^{-}, s^{-}, o^{-}, w^{-}$, wy-,od-, which form Polish equivalents of French verb tomber, and each of them adds further semantic information to the meaning of verb basis.

\section{INDEX}

Mots-clés : invariant, base verbale, préverbe polonais, éléments de sens, information sémantique

Keywords : invariant, verbal basis, Polish preverb, meaning component, semantic information 
AUTEUR

JOANNA CHOLEWA

Université de Bialystok, Pologne 